\title{
Chronological Changes in Neutrophil/lymphocyte Ratio in Advanced Gastric Cancer Patients Treated with Nivolumab: a Report of Nine Cases
}

\author{
Naohiko Nakamura*, Shinichi Kinami, Jun Fujita, Daisuke Kaida, Yasuto \\ Tomita, Takashi Miyata, Hideto Fujita, Hiroyuki Takamura, Nobuhiko Ueda, \\ Takeo Kosaka
}

\begin{abstract}
Background: Nivolumab has been approved for use in advanced gastric cancer (GC) after third-line chemotherapy in Japan. However, it remains difficult to predict favorable nivolumab response before treatment. Methods: We evaluated the clinical course with a focus on the chronological changes in neutrophil/lymphocyte ratio (NLR) throughout the chemotherapy and assessed the relationship between nivolumab response and chronological changes in NLR before nivolumab administration. Results: We experienced nine cases who received nivolumab monotherapy for unresectable advanced or postoperative recurrent GC. Nivolumab was used as third-line chemotherapy in all patients, and partial response (PR) and stable disease (SD) were observed in two patients each. Nivolumab treatment resulted in progressive disease (PD) in five patients. In patients with PR or SD, changes in the NLR tended to correspond to the response of target metastatic lymph nodes to first- and second-line chemotherapy. In the four cases with PR or SD following nivolumab, $\triangle \mathrm{NLR}_{\text {responses }}$ that was the difference in the degree of decline during the most effective pretreatment chemotherapy were $1.39,0.73,1.62$, and 1.22. However, the patients with PD showed lower $\Delta \mathrm{NLR}_{\text {responses }}$, at $0.66,0.66,0.25,0.13$, and -0.05 in the five cases. Mean $\triangle \mathrm{NLR}_{\text {responses }}$ in the patients with PR or SD and patients with PD were 1.17 and 0.33 , respectively $(\mathrm{P}=0.0008)$. Conclusions: We experienced nine $\mathrm{GC}$ cases treated with nivolumab and assessed the association between chronological NLR changes throughout chemotherapy and tumor response to nivolumab. Changes in NLR during pretreatment chemotherapy might predict tumor response to nivolumab monotherapy in patients with advanced GC.
\end{abstract}

Keywords: Gastric cancer- nivolumab- neutrophil/lymphocyte ratio

Asian Pac J Cancer Prev, 21 (10), 2955-2960

\section{Introduction}

Immune checkpoint inhibitors (ICIs) are now widely administered for the treatment of many kinds of cancer. The 2017 ATTRACTION-2 study showed that nivolumab monotherapy was more effective in patients with advanced gastric cancer (GC) (Kang et al., 2017). Although the response rate in nivolumab monotherapy was $11.2 \%$ in that study, it remains difficult to predict favorable nivolumab response before treatment. Recently, Ogata et al. reported that the neutrophil/lymphocyte ratio (NLR) may be an effective prognostic factor in patients with advanced GC receiving nivolumab treatment (Eisenhauer et al., 2009). In addition, there has been increasing interest in the association between the NLR and the clinical outcomes of upper gastrointestinal cancers. With regard to prognostic outcomes after gastrectomy in patients with $\mathrm{GC}$, a retrospective analysis showed an association between a high NLR and poor survival (el-Hag and Clark, 1987). However, detailed chronological changes in the NLR have not been assessed in patients with advanced GC treated with multiple anticancer agents including nivolumab. An assessment of chronological changes in NLR throughout chemotherapy, especially during the nivolumab pretreatment period, might help to predict nivolumab monotherapy response in patients with advanced GC. We report our experience with nine patients with GC treated with nivolumab. We evaluated the clinical course with a focus on the chronological changes in NLR throughout the chemotherapy and assessed the relationship between nivolumab response and chronological changes in NLR before nivolumab administration. 


\section{Materials and Methods}

We retrospectively analyzed the data of nine patients with unresectable advanced or postoperative recurrent GC who received nivolumab treatment at Kanazawa Medical University Hospital between September 2017 and December 2019. Data of clinical parameters such as sex, age, clinical course, and anticancer agents used for GC treatment were extracted from our hospital database. We calculated the NLR, defined as the ratio of neutrophil to lymphocyte counts in the white blood cell differential, from the results of blood examinations throughout the treatment. Using imaging studies, the patients were clinically staged based on the 7th edition of the American Joint Committee on Cancer (Ferlay et al., 2015) according to the depth of tumor invasion (T), extent of lymph node metastasis $(\mathrm{N})$, and presence of distant metastasis (M). Responses of the target lesions to chemotherapy were classified according to the Response Evaluation Criteria in Solid Tumors (RECIST) guidelines (Kang et al., 2017). NLRresponse was defined as the NLR for the most effective response by RECIST obtained in first- or second-line chemotherapy regimens before nivolumab administration. NLRpre was defined as the NLR at initiation of the most effective regimen. $\Delta$ NLRresponse was defined as the difference in the degree of decline from NLRpre to NLRresponse. Data were expressed as mean ( \pm standard deviation). Continuous variables were compared using Student's t-tests. All statistical analyses were performed using JMP version 8.0 (SAS Institute, Cary, NC, USA).

We obtained informed consent from the patients. This study was approved by the Medicine Ethics Committee of Kanazawa Medical University.

\section{Results}

Patient characteristics and tumor response to nivolumab

Nine patients in this study received nivolumab monotherapy; their characteristics are shown in Table 1. Mean patient age was 70.0 years, and $77.8 \%$ $(n=7)$ were men. Among the nine patients, four and five had postoperative recurrent and unresectable advanced GC, respectively. Seven patients underwent S-1-based chemotherapy as first-line chemotherapy, and seven patients underwent ramucirumab and/or (nab-) paclitaxel as second-line chemotherapy. Nivolumab was used as third-line chemotherapy in all nine patients. Following nivolumab monotherapy, partial response (PR) and stable disease (SD) were observed in two patients each, while five patients had progressive disease (PD).

\section{Chronological changes in NLRs and target lesions throughout chemotherapy}

In the two patients with PR following nivolumab treatment, the NLR decreased corresponding to the reduction in the diameters of the target metastatic lymph nodes in first- and second-line chemotherapy. However, the NLR at nivolumab initiation increased in both patients (Figure 1: Cases 1 and 2). The patients with SD following nivolumab treatment (Figure 1: Cases 3 and 4)

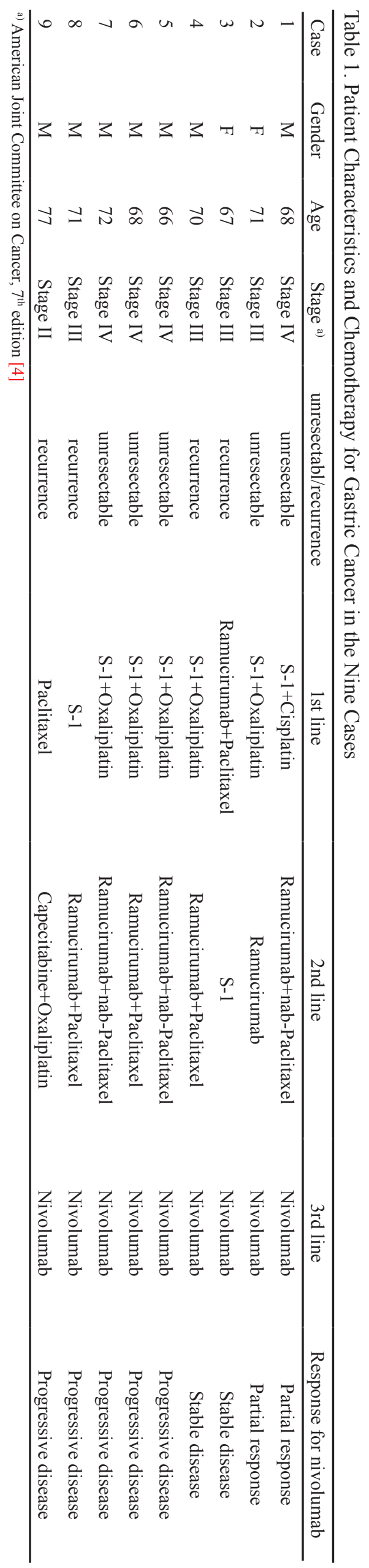


Table 2. $\triangle$ NLR $_{\text {resnonse }}$ According to Tumor Response to Nivolumab in the Nine Cases

\begin{tabular}{lccc}
\hline Case & Response for nivolumab & $\Delta \mathrm{NLR}_{\text {response }}$ & ${\text { Mean } \Delta \mathrm{NLR}_{\text {response }}}$ \\
\hline 1 & $\mathrm{PR}$ & 1.39 & $1.17 \pm 0.17$ \\
2 & $\mathrm{PR}$ & 0.73 & \\
3 & $\mathrm{SD}$ & 1.62 & \\
4 & $\mathrm{SD}$ & 1.22 & \\
5 & $\mathrm{PD}$ & -0.05 & $0.33 \pm 015$ \\
6 & $\mathrm{PD}$ & 0.66 & \\
7 & $\mathrm{PD}$ & 0.25 & \\
8 & $\mathrm{PD}$ & 0.66 & \\
9 & $\mathrm{PD}$ & 0.13 & \\
9 & $\mathrm{Mean} \Delta \mathrm{NLR}_{\text {response }} \pm$ standard deviation in patients with PR or SD \\
$(\mathrm{n}=4)$ and with PD ( $=5$ ); Partial response, PR; stable disease, SD; \\
progressive disease, PD
\end{tabular}

showed decreased NLR corresponding to the regression of target metastatic lymph nodes following ramucirumab + paclitaxel administration. In contrast, the five patients with PD did not tend to show significant chronological changes in NLR corresponding to tumor response or significant decreases in NLR even if effective disease control was obtained following the first- or second-line chemotherapy (Figure 1: Cases 5-9).

Correlation between $\triangle N L R_{\text {response }}$ and tumor response for nivolumab

$\triangle \mathrm{NLR}_{\text {responses }}$ in the two cases with PR following nivolumab were 1.39 and 0.73 , while that in the two cases with SD were 1.62 and 1.22. However, the patients with PD showed lower $\Delta \mathrm{NLR}_{\text {responses }}$, at $0.66,0.66,0.25,0.13$, and -0.05 in the five cases (Table 2). Mean $\triangle$ NLR $_{\text {responses }}$ in the patients with $\mathrm{PR}$ or SD and patients with PD were 1.17 and 0.33 , respectively $(\mathrm{P}=0.0008)$. The patients who achieved effective control with nivolumab therapy had a
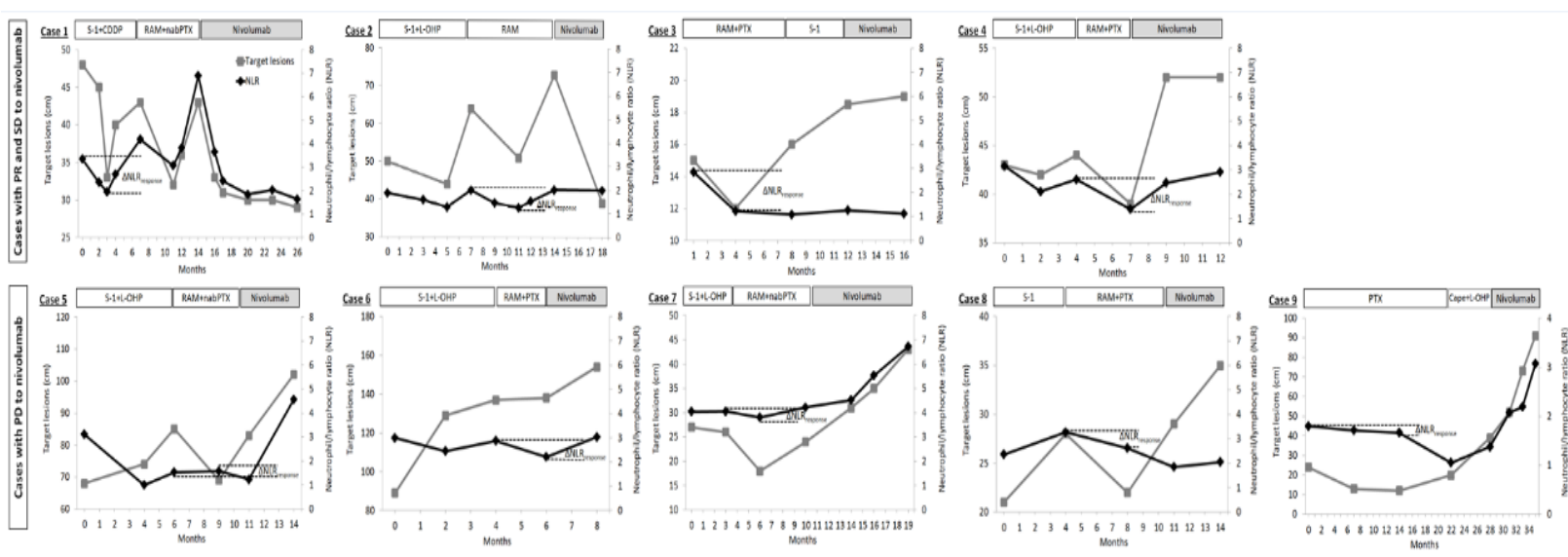

Figure 1. Gastric Cancer Treatments and Chronological Changes in NLR and Target Lesions in the Nine Cases. Case 1 and 2 showed partial response (PR) to nivolumab. Case 3 and 4 showed stable disease (SD) to nivolumab. Case 5, 6, 7, 8, and 9 showed progressive disease (PD) to nivolumab. Neutrophil/lymphocyte ratio, NLR; cisplatin, CDDP; ramucirumab, RAM; (nab-) paclitaxel, (nab-) PTX; oxaliplatin, L-OHP; capecitabine, Cape

Table 3. Previous Case Reports in GC Patients Treated with Nivolumab

\begin{tabular}{|c|c|c|c|c|c|}
\hline References & Age & Sex & Diagnosis & Chemotherapy prior to nivolumab & $\begin{array}{l}\text { Response to } \\
\text { nivolumab }\end{array}$ \\
\hline $\begin{array}{l}\text { Namikawa T, et al. } 2018 \\
{[21]}\end{array}$ & 77 & $\mathrm{M}$ & $\begin{array}{l}\text { GC with liver metastases } \\
\text { after surgical resection }\end{array}$ & S-1+Oxaliplatin & $\mathrm{CP}$ \\
\hline \multirow[t]{2}{*}{$\begin{array}{l}\text { Togasaki K, et al. } 2018 \\
\text { [22] }\end{array}$} & 71 & M & GC with liver metastases & $\begin{array}{l}\text { Trastuzumab+Capeciabine }+ \text { Cysplatin/ } \\
\text { Ramucirumab+Paclitaxel }\end{array}$ & PR \\
\hline & 79 & M & $\begin{array}{l}\text { GC with para-aortic lymph } \\
\text { node metastasis }\end{array}$ & S-1+Cisplatin/Paclitaxel & PR \\
\hline $\begin{array}{l}\text { Satoyoshi R, et al. } 2019 \\
\text { [23] }\end{array}$ & 88 & M & $\begin{array}{l}\text { GC with liver metastases } \\
\text { after surgical resection }\end{array}$ & None & PR \\
\hline $\begin{array}{l}\text { Kashima S, et al. } 2019 \\
\text { [24] }\end{array}$ & 25 & M & $\begin{array}{l}\text { GEC with metastatic } \\
\text { lymph nodes after surgical } \\
\text { resection }\end{array}$ & Trastuzumab + Capeciabine + Cysplatin & $\mathrm{CR}$ \\
\hline Toyota S, et al. 2020 [25] & 75 & M & $\begin{array}{l}\text { GC with peritoneal } \\
\text { metastases }\end{array}$ & $\begin{array}{l}\text { S-1+Oxaliplatin / } \\
\text { Ramucirumab+Paclitaxel }\end{array}$ & PR \\
\hline \multirow[t]{2}{*}{ Tirino G, et al. 2020 [26] } & 78 & $\mathrm{~F}$ & GC with liver metastases & mFOLFOX-6 /Ramucirumab+Paclitaxel & $\mathrm{CR}$ \\
\hline & 82 & M & $\begin{array}{l}\text { GEC with liver metastases } \\
\text { after surgical resection }\end{array}$ & mFOLFOX-6 /Ramucirumab+Paclitaxel & PR \\
\hline
\end{tabular}

Partial response, PR; stable disease, SD; progressive disease, PD; GEC, gastroesophageal cancer 
significantly higher $\Delta$ NLRresponse.

\section{Discussion}

We reported our experience with nine patients with GC receiving nivolumab monotherapy. We examined the chronological changes in NLR throughout chemotherapy and assessed the relationship between NLR changes during pretreatment chemotherapy and tumor responses to nivolumab monotherapy.

$\mathrm{GC}$ is the fifth most common cancer and the third leading cause of cancer mortality worldwide (Kashima et al., 2019). Although some chemotherapy regimens have been shown to improve the survival outcomes of patients with advanced GC, the prognosis remains poor and hence further therapeutic development is needed. In this context, nivolumab, a fully human IgG4 monoclonal antibody against programmed death-1 (PD-1), has been approved for use in advanced GC after third-line chemotherapy in Japan and is expected to improve the prognosis of patients with advanced GC. However, it remains difficult to adjust the indication for nivolumab use and predict tumor response before nivolumab administration. Moreover, we are now facing challenges in choosing third-line chemotherapeutic agents between nivolumab or trifluridine/tipiracil (also known as TAS102), a novel oral combination cytotoxic drug that has recently been reported to significantly improve overall survival in patients with advanced GC (Le et al., 2015). Thus, it is important to determine clinical parameters that can predict tumor response to nivolumab monotherapy.

The ATTRACTION-2 study reported a response rate of $11.2 \%$ and a median overall survival of 5.26 months (Edge and Compton, 2010). The present study retrospectively analyzed nine patients who received nivolumab monotherapy, two of whom $(22.2 \%)$ showed PR and better prognosis. We excluded six patients in whom nivolumab monotherapy was initiated but was stopped after fewer than three administrations owing to the deterioration of their general condition because of GC progression. Because nivolumab is used as third- or laterline treatment for advanced GC and patients tend to show worse performance status (PS), the patients should show sufficient PS for the continuation of nivolumab treatment. All nine patients in this study had Eastern Cooperative Oncology Group performance status (ECOG-PS) scores of $0-1$; thus, nivolumab could be continuously administered until imaging assessment of tumor response. Before nivolumab administration, eight patients were treated with S-1 - or ramucirumab-based chemotherapy as first- or second-line therapies. These treatments reduced the target lesion diameter in four and seven patients, respectively. The tumor response based on the RECIST criteria in pretreatment chemotherapy was not associated with the response to nivolumab monotherapy. List of previous case reports in GC patients treated with nivolumab was shown Table 3. GC or gastroesophageal cancer patients underwent nivolumab monotherapy for liver metastases or lymph node metastases, and obtained good response to nivolumab. However, the chronological changes in
NLR throughout chemotherapy were not assessed in these case reports.

This case report implied that $\triangle$ NLRresponse, defined as changes in NLR during pretreatment chemotherapy, might predict tumor response to nivolumab monotherapy in patients with advanced GC. Recent reports have indicated better prognosis in advanced GC patients with a low NLR before the first cycle of nivolumab or a reduction in NLR during nivolumab monotherapy (Eisenhauer et al., 2009; Nakamura et al., 2019). However, chronological changes in NLR during pretreatment chemotherapy have not been assessed as a predictor for tumor response in nivolumab monotherapy. Studies on ICIs have reported the association between their efficacy and PD-L1 or PD-L2 expression, mutation burden, and deficient mismatch repair (dMMR) (Namikawa et al., 2018; Ohmura et al., 2020). In addition, it was indicated that the expression levels of LAG-3 and OX40 on T cells correlated with the efficacy of nivolumab therapy (Ota et al., 2020). Compared to these parameters that could involve the antitumor mechanism of nivolumab, the NLR is a simpler index that could be calculated in routine examinations and used as a non-invasive biomarker. Elevated NLR is reportedly related to the poor prognosis of GC, colorectal, and lung cancer (Petrie et al., 1985; Shimada et al., 2010). We also previously showed an association between a high NLR and the presence of peritoneal metastasis in patients with advanced GC (Shimada et al., 2010). The mechanism underlying the association of $\triangle$ NLRresponse with tumor response to nivolumab remains unclear. Because the NLR can be considered an index reflecting systemic inflammatory response (Shitara et al., 2018; Tirino et al., 2020) and a decreased number of neutrophils promote lymphocyte activity (Tirino et al., 2010; Togasaki et al., 2018), cytotoxic or molecular targeted agents such as those administered during pretreatment chemotherapy for nivolumab could suppress systemic inflammation through tumor regression; the neutrophil count may result in a better response to nivolumab monotherapy due to increasing levels of activated lymphocytes.

This study has several limitations. This study has a retrospective design and a small sample size of only nine cases from a single institution. We report only an observed tendency regarding the relationship between changes in NLR and tumor response to nivolumab monotherapy. Thus, further retrospective or prospective cohort studies with sufficient sample sizes are needed to confirm the predictive value of the NLR for tumor response to nivolumab monotherapy.

In conclusion, we experienced nine cases administered nivolumab monotherapy for unresectable advanced or postoperative recurrent GC and assessed the association between chronological NLR changes throughout chemotherapy and tumor response to nivolumab. Changes in NLR during pretreatment chemotherapy might predict tumor response to nivolumab monotherapy in patients with advanced GC. 


\section{Acknowledgements}

Not applicable.

\section{List of abbreviations}

Immune checkpoint inhibitors: ICIs; gastric cancer: GC; neutrophil/lymphocyte ratio: NLR; partial response: PR; stable disease: SD; progressive disease: PD; programmed death-1: PD-1; performance status: PS

\section{Declarations}

Availability of data and materials: All data are available without restriction. Researchers can obtain data by contacting the corresponding author.

\section{Consent for publication}

We obtained informed consent from the patients.

\section{Ethics approval and consent to participate}

This study was approved by the Medicine Ethics Committee of Kanazawa Medical University. The research reported in this paper was in compliance with the Helsinki Declaration.

\section{Authors' contributions}

NN, SK and TK designed the study. NN, YT, and TM performed data acquisition, data analysis, and interpretation. NN and SK prepared the manuscript. SK, HF, HT, NU, and TK revised paper critically. All authors read and approved the final manuscript.

\section{Competing interests}

The authors declare no competing interest in connection with this study.

\section{References}

Edge SB, Compton CC (2010). The American Joint Committee on Cancer: the 7th edition of the AJCC cancer staging manual and the future of TNM. Ann Surg Oncol, 17, 1471-4.

Eisenhauer EA, Therasse P, Bogaerts J, et al (2009). New response evaluation criteria in solid tumours: revised RECIST guideline (version 1.1). Eur J Cancer, 45, 228-47.

el-Hag A, Clark RA (1987). Immunosuppression by activated human neutrophils. Dependence on the myeloperoxidase system. J Immunol, 139, 2406-13.

Ferlay J, Soerjomataram I, Dikshit R, et al (2015). Cancer incidence and mortality worldwide: sources, methods and major patterns in GLOBOCAN 2012. Int J Cancer, 2015 , 359-86.

Kang YK, Boku N, Satoh T, et al (2017). Nivolumab in patients with advanced gastric or gastro-oesophageal junction cancer refractory to, or intolerant of, at least two previous chemotherapy regimens (ONO-4538-12, ATTRACTION-2): a randomised, double-blind, placebo-controlled, phase 3 trial. Lancet, 390, 2461-71.

Kashima S, Tanabe H, Tanino M, et al (2019). Lymph node metastasis from gastroesophageal cancer successfully treated by Nivolumab: A Case Report of a Young Patient. Front Oncol, 9, 1375.

Le DT, Uram JN, Wang H, et al (2015). PD-1 blockade in tumors with mismatchrepair deficiency. $N$ Engl $J$ Med, 372, 2509-20.
Nakamura N, Kinami S, Fujii Y, et al (2019). The neutrophil/ lymphocyte ratio as a predictor of peritoneal metastasis during staging laparoscopy for advanced gastric cancer: a retrospective cohort analysis. World J Surg Oncol, 25, 108.

Namikawa T, Ishida N, Tsuda S, et al (2018). Successful treatment of liver metastases arising from early gastric cancer achieved clinical complete response by nivolumab. Surg Case Rep, 4, 71.

Ogata T, Satake H, Ogata M, et al (2018). Neutrophil-tolymphocyte ratio as a predictive or prognostic factor for gastric cancer treated with nivolumab: a multicenter retrospective study. Oncotarget, 9, 34520-7.

Ohmura H, Yamaguchi K, Hanamura F, et al (2020). OX40 and LAG3 are associated with better prognosis in advanced gastric cancer patients treated with anti-programmed death-1 antibody. Br J Cancer, 2020. doi: 10.1038/s41416-0200810-1

Ota Y, Takahari D, Suzuki T, et al (2020). Changes in the neutrophil-to-lymphocyte ratio during nivolumab monotherapy are associated with gastric cancer survival. Cancer Chemother Pharmacol, 2020, doi: 10.1007/s00280019-04023-w.

Petrie HT, Klassen LW, Kay HD (1985). Inhibition of human cytotoxic $\mathrm{T}$ lymphocyte activity in vitro by autologous peripheral blood granulocytes. J Immunol, 134, 230-4.

Satoyoshi R, Muto O, Masuda A, et al (2019). A case of gastric cancer with delayed onset of tumor reduction effect by nivolumab therapy. Clin J Gastroenterol, 12, 15-9.

Shimada H, Takiguchi N, Kainuma O, et al (2010). High preoperative neutrophil-lymphocyte ratio predicts poor survival in patients with gastric cancer. Gastric Cancer, 13, 170-6.

Shimada H, Takiguchi N, Kainuma O, et al (2010). High preoperative neutrophil-lymphocyte ratio predicts poor survival in patients with gastric cancer. Gastric Cancer, 13, 170-6.

Shitara K, Doi T, Dvorkin M, et al (2018) Trifuridine/tipiracil versus placebo in patients with heavily pretreated metastatic gastric cancer (TAGS): a randomised, double-blind, placebocontrolled, phase 3 trial. Lancet Oncol, 19, 1437-48.

Takahashi M (2016). Genetic mutation accumulation and clinical outcome of immune checkpoint blockade therapy. Gan To Kagaku Ryoho, 43, 678-82 (in Japanease).

Tirino G, Petrillo A, Pompella L, et al (2020). Durable complete radiological response to Nivolumab in two heavily pretreated western elderly patients with metastatic gastric Cancer: A Case Report. Front Oncol, 10, 130.

Togasaki K, Sukawa Y, Kanai T, Takaishi H (2018). Clinical efficacy of immune checkpoint inhibitors in the treatment of unresectable advanced or recurrent gastric cancer: an evidence-based review of therapies. Onco Targets Ther, 11, 8239-50.

Tomita M, Shimizu T, Ayabe T, Yonei A, Onitsuka T (2011). Preoperative neutrophil to lymphocyte ratio as a prognostic predictor after curative resection for non-small cell lung cancer. Anticancer Res, 31, 2995-8.

Toyota S, Orita H, Fukuyama Y, et al (2020). Successful conversion surgery following chylous ascites after nivolumab for advanced gastric cancer. In Vivo, 34, 583-5.

Walsh SR, Cook EJ, Goulder F, Justin TA, Keeling NJ (2005). Neutrophil-lymphocyte ratio as a prognostic factor in colorectal cancer. J Surg Oncol, 91, 181-4.

Wang L, Zhang Q, Ni S, et al (2018). Programmed death-ligand 1 expression in gastric cancer: correlation with mismatch repair deficiency and HER2-negative status. Cancer Med, 7, 2612-20.

Zahorec R (2001). Ratio of neutrophil to lymphocyte counts-- 
Naohiko Nakamura et al

rapid and simple parameter of systemic inflammation and stress in critically ill. Bratisl Lek Listy, 102, 5-14.19.

\section{(c) (i) (8)}

This work is licensed under a Creative Commons AttributionNon Commercial 4.0 International License. 